\title{
The evolution of advanced techniques for the management of symptomatic aortic stenosis in the elderly population: conventional surgical management vs transcatheter valve implantation
}

\author{
${ }^{1} \mathrm{M}$ Jahangiri, ${ }^{2} \mathrm{AP}$ Kappetein, ${ }^{3} \mathrm{M}$ van Geldorp, ${ }^{4} \mathrm{AJ}$ Bogers \\ ${ }^{1}$ Professor of Cardiac Surgery, St George's Healthcare NHS Trust, London, UK; ${ }^{2}$ Associate Professor of Cardiac Surgery; ${ }^{3}$ PhD Student; \\ ${ }^{4}$ Professor and Director of Cardiothoracic Surgery, Department of Cardio-Thoracic Surgery, Erasmus MC Rotterdam, The Netherlands
}

\begin{abstract}
The shifting age demographic of the adult population has affected every area of contemporary medical and surgical practice. Many more people are living well, not just into their 70s but into their 80 s and beyond. Their expectations of treatment for every illness have shifted markedly upwards at the same time. Despite the decline in cases of rheumatic fever in Westernised populations in recent times, the ageing population has led to no decline in the prevalence of valvular aortic stenosis. This is now realised to be an active pro-inflammatory disease, rather than a degenerative process. Thus the condition has remained in the mainstream and continues to be responsible for considerable morbidity, hospitalisation and mortality among the elderly and very elderly.
\end{abstract}

Management has always been based on the triage of cases for direct intervention to the valve by surgery. Just as expectations have risen from patients, the techniques, application and monitoring of cardiac surgery have also made huge strides forward to meet this aspiration. More and more, surgeons are routinely asked to consider procedures in frailer, more elderly patients with more severe disease and co-morbidity. Managing the stenosis is rarely the only issue confronting the operating surgeon. Attempts to provide alternatives to open valve replacement surgery on cardiopulmonary bypass have now emerged. These are based around the transcutaneous placement of a valve prosthesis. While these technologies were initially highly selective in their application, they have now reached a stage to be compared with contemporary standards of cardiac surgical practice. In this debate we have invited two international experts from the fields of cardiac surgery (Professor Jahangiri) and interventional cardiology (Professor Kappetein and colleagues) to take deliberately opposing positions on the evolving management of valvular aortic stenosis in the very elderly. We have asked them to try to consider the strengths of each route. Both approaches provide options for patients who only a few years ago might have been regarded as essentially untreatable.

KEYWORDS Aortic valve stenosis, aortic valve surgery, transcatheter aortic valve implantation

DECLARATION OF INTERESTS No conflict of interests declared.

\author{
Correspondence to \\ $M$ Jahangiri, \\ Department of Cardiothoracic \\ Surgery, St George's Healthcare \\ NHS Trust, Blackshaw Road, \\ London SWI7 OQT, UK
}

tel. +44 (0)20 87253565

e-mail fwilliam@sgul.ac.uk

AP Kappetein,
Department of Cardio-Thoracic
Surgery, Room BD 569, Erasmus
MC, PO Box 2040,
3000 CA Rotterdam,
The Netherlands
tel.+3I 107035412
e-mail a.kappetein@erasmusmc.nl

\section{$\mathrm{TAVI}$ and surgery in high-risk aortic valve patients}

\section{Jahangiri}

With the advent of transcatheter aortic valve implantation (TAVI) and its recent expansion, ${ }^{1,2}$ the number of patients being referred for the management of aortic valve (AV) disease has increased. It is also perceived that the less invasive TAVI is associated with better outcomes. Previously medically managed patients with AV disease are now being referred for intervention. A European heart survey on valvular heart disease showed that $33 \%$ of patients with severe symptomatic aortic stenosis (AS) did not undergo surgery. ${ }^{3}$ Other surveys have shown that approximately half of patients with severe AS did not undergo surgery, where the operative risks were thought to be $5-12 \% .{ }^{4}$ This may be due to a lack of knowledge of referring physicians about surgical risk scoring and the results of surgery. Bach and colleagues reported that among 30 patients who were thought to have prohibitive risks for surgery, calculated operative risk was less than $5 \%$ in 11 and less than $10 \%$ in 17 of them and only half were evaluated by surgeons. ${ }^{4}$

The advent of TAVI has created a resurgence of interest in the multidisciplinary meeting as a process. There are 
several reports of outcomes of patients who have undergone TAVI. 2.5

The risk of aortic valve replacement (AVR) in octogenarians is reported to be approximately $5-8 \% .{ }^{6}$ In the national database of cardiac surgery over a six-year period, this figure was approximately $5.5 \%$, with the overall risk of stroke less than $2 \%{ }^{1,2}$

To this date, data from various registries from both the UK and other surveys within Europe ${ }^{7}$ show comparable mortality and length of hospital stay for both TAVI and surgical AVR. However, the risk of stroke in TAVI (6-8\%) is significantly higher than in patients undergoing surgical AVR (2\%). In a recent study, cerebral ischaemia was assessed by neurological testing and serial cerebral diffusion-weighted magnetic resonance imaging in patients who underwent TAVI compared with AVR. ${ }^{8}$ The authors detected clinically silent new foci of restricted diffusion on magnetic resonance imaging in $84 \%$ of TAVI patients with a multiple dispersed pattern suggesting cerebral embolisation. This may be due to the showering of emboli from the aorta, particularly the arch, or the dislodgement of aortic leaflet plaques. If emboli from the arch play a more significant role, it may be that transapical delivery systems potentially reduce the risks of embolisation. Some have suggested the use of distal protection devices to prevent emboli reaching the brain. ${ }^{9}$ However, the potential benefits of these future devices have to be balanced against their own invasiveness, prolongation of the procedure and existing results of surgery.

Furthermore, the need for pacemaker implantation following TAVI, especially with some of the devices, such as CoreValve, is as high as $25 \%$ in the TAVI population, compared with nearly zero in surgical patients. Additionally, up to half of TAVI patients develop mild to moderate aortic regurgitation following the procedure. ${ }^{79}$

Although data from registries are not randomised and therefore subject to the inherent deficiencies of nonrandomised studies, they reflect 'real-world' practice. Some of the data from the 'real-world' practice include outcomes of all high-risk patients discussed at multidisciplinary meetings for consideration of TAVI. There

\section{REFERENCES}

I Grube E, Buellesfeld L, Mueller R et al. Progress and current status of percutaneous aortic valve replacement: results of three device generations of the CoreValve revalving system. Circ Cardiovasc Intervent 2008; I: I67-75. doi:I0.I I6I/CIRCINTERVENTIONS.108.8I9839

2 Piazza N, Grube E, Gerckens U et al. Procedural and 30-day outcomes following transcatheter aortic valve implantation using the third generation ( $18 \mathrm{Fr}$ ) corevalve revalving system: results from the multicentre, expanded evaluation registry I-year following CE mark approval. Eurolntervention 2008; 4:242-9.

3 lung B, Cachier A, Baron G et al. Decision-making in elderly patients with severe aortic stenosis: why are so many denied surgery? Eur Heart J 2005; 26:27|4-20. doi:I0.1093/eurheartj/ehi47| are as yet no randomised studies to compare the outcome of patients undergoing surgical AVR and TAVI, particularly the quality of life following treatment. The results of the PARTNER trial in the US, a large randomised trial comparing TAVI with surgical AVR in patients suitable for both, is pending.

The new techniques of surgery, including mini-AVR and sutureless valves, deserve attention. The mini-AVR is performed via a limited sternotomy or a small rightsided lateral thoracotomy. Several large studies have shown excellent outcomes, especially shorter time to extubation and improved pulmonary function. ${ }^{10}$ The future sutureless valves will also allow shorter cross clamp and cardiopulmonary bypass times.

An expanding and promising use of TAVI is in patients with previous cardiac surgery, be it coronary artery bypass graft or valvular heart surgery where there are patent grafts or a malfunctioning tissue prosthesis present, the so-called valve-in-valve procedure. The risk of re-do surgery, particularly in the elderly, in this cohort can be high. However, the age of the patient and complexity of the operation should be weighed against the potential durability and complications of the transcatheter valve. The notion that all patients who have undergone previous cardiac surgery no longer qualify for re-do operations is not correct.

Transcatheter AV implantation is a promising and important development in the field of cardiovascular intervention. However, its current reported mortality, significantly higher stroke rate, mild to moderate aortic regurgitation and high rate of pacemaker implantation should be considered before its wider application, especially to a younger population. This is particularly important considering the very good results of surgery in the UK for $\mathrm{AV}$ disease. Furthermore, the referring physicians should be made aware of the current very good results of surgery in the elderly.

Transcatheter AV implantation is a promising development. Its use must be considered in the setting of a multidisciplinary meeting where interventional and non-interventional cardiologists and cardiac surgeons are present.

4 Bach DS, Cimino N, Deeb GM. Unoperated patients with severe aortic stenosis. J Am Coll Cardiol 2007; 50:2018-9. doi:10.1016/j. jacc.2007.08.0II

5 Webb JG, Pasupati S, Humphries K et al. Percutaneous transarterial aortic valve replacement in selected high-risk patients with aortic stenosis. Circulation 2007; II6:755-63. doi:I0.II6I/ CIRCULATIONAHA.107.698258

6 Fruitman DS, MacDougall CE, Ross DB. Cardiac surgery in octogenarians: can elderly patients benefit? Quality of life after cardiac surgery. Ann Thorac Surg 1999: 68:2129-35. doi:10.1016/ S0003-4975(99)00818-8 
7 Thomas M, Schymik G, Walther T et al. Thirty-day results of the SAPIEN aortic bioprosthesis European Outcome (SOURCE) Registry: a European registry of transcatheter aortic valve implantation using the Edwards SAPIEN valve. Circulation 2010; I22:62-9. doi: I0.I I6I/CIRCULATIONAHA.109.907402

8 Kahlert P, Knipp SC, Schlamann M et al. Silent and apparent cerebral ischemia after percutaneous transfemoral aortic valve implantation: a diffusion-weighted magnetic resonance imaging study. Circulation 2010; I2I:870-8. doi: I0.I I6I/CIRCULATIONAHA.109.855866
9 Charalambous N, Jahnke T, Bolte $\mathrm{H}$ et al. Reduction of cerebral embolization in carotid angioplasty: an in-vitro experiment comparing 2 cerebral protection devices. J Endovasc Ther 2009; 16:16I-7. doi:10.1583/08-2355.I

10 Mihaljevic T, Cohn LH, Unic D et al. One thousand minimally invasive valve operations: early and late results. Ann Surg 2004; 240:529-34. doi:I0.1097/0I.sla.0000137|4I.55267.47

\title{
Promising results with transcatheter valve implantation, but more data are needed
}

\author{
AP Kappetein, M van Geldorp, AJ Bogers
}

Aortic valve stenosis (AS) is the most frequent valvular heart disease in the adult population in developed countries, affecting approximately $2-4 \%$ of people over 65 years of age. ${ }^{1,2}$ Increased life expectancy has resulted in a growing elderly population and, consequently, an increase in the number of patients with aortic valve disease. This results in approximately three million people with AS in Europe alone. One in five will eventually progress to symptomatic AS, representing 600,000 patients.

Once symptoms appear, the prognosis is very poor. Median survival averages only two to five years after symptom onset of angina, syncope and heart failure. ${ }^{3}$ Medical therapy is unlikely to modify the course of the disease, especially once symptoms or left ventricular (LV) dysfunction become manifest. Balloon aortic valvuloplasty has only a limited role in the treatment of AS, as the results are not long-lasting. ${ }^{4}$ Surgical aortic valve replacement $(A V R)$ is the reference treatment and guidelines on valvular heart disease stress the need for surgical aortic valve replacement (SAVR) once symptoms develop or in case of impaired LV function (level of evidence grade I).

Despite these well-established guidelines, one in every three patients with symptomatic AS is not offered surgery, mostly because of age, LV dysfunction and co-morbidities. ${ }^{7,8}$ Elderly patients and patients suffering from severe co-morbidities face higher operative risks and this definitely underscores an unmet clinical need. Undoubtedly this reality and patients' and physicians' preferences for less invasive strategies have fuelled the ongoing interest in developing minimally invasive therapies. Minimised access AVR was established, which decreased surgical trauma but still required extracorporeal circulation with its associated complications.

Transcatheter aortic valve implantation (TAVI) met the need for a less invasive solution to treat severe aortic stenosis in the high-risk elderly population, in whom few options were available. Feasibility studies validated the proof of concept. ${ }^{9} 10$ There are now two different TAVI systems clinically available with CE mark approval since 2007: Edwards SAPIEN valve (Edwards Life Sciences, Irvine, California) and CoreValve (Medtronic, Minneapolis). Numerous single-centre and multi-centre observational registries followed with dazzling speed, suggesting the safety and efficacy of the TAVI technology. ${ }^{11,12}$

The availability of both transfemoral and transapical approaches increased the number of those patients, in comparison with the use of the transfemoral approach alone. Although the mortality rate with TAVI was higher in earlier reports, the 30 -day mortality of around $8 \%$ in patients with high or prohibitive operative risk appears promising and resembles short-term outcome in highrisk cohorts in the surgical literature. ${ }^{13-16}$ The SOURCE registry, which has the largest patient population, reported a 30 -day mortality of $8.5 \%$, with respective mortality rates of $6.3 \%$ and $10.3 \%$ in the transfemoral and transapical groups. ${ }^{17}$

Numerous studies have documented a dramatic reduction in the left ventricle-aortic gradient and a marked increase in the valve area. On the basis of postoperative echocardiograms, the effective orifice area of the transcatheter prosthesis is as good as, if not better than, that of valves placed in open surgery. ${ }^{17}$ The only concern is a higher incidence of paravalvular leakage. A less than grade $2+$ regurgitation seems to be well tolerated without heart failure or haemolysis, but the long-term effect remains unclear.

This innovation in cardiovascular therapeutics has been a collaborative effort by both interventional cardiologists and surgeons and has resulted in a rapid acceptance of TAVI in Europe. The TAVI technology comes with its own complications: vascular injury, stroke, cardiac injury, malposition, coronary obstruction, cardiac perforation, aortic regurgitation and heart block. The non-uniformity in presenting respective data makes comparison of results from different centres hazardous and impractical. ${ }^{18}$ The Valvular Academic Research Consortium, a Food and Drug Administration-approved collaboration 
between academic research organisations and professional societies in the US and Europe is an initiative to generate a consensus statement on TAVIrelated definitions aiming to create order and uniformity, making data more prone to analysis and comparison.

Technical refinements and commercial entrepreneurship have made the technology accessible to many centres worldwide. This might pose future implications, especially in the current era where randomised trials with TAVI are strikingly lacking.

Randomised controlled trials comparing TAVI with SAVR with longer follow-up are the next step and will help to better define the safety, durability and, subsequently, indications of the technique and the respective places of transfemoral and transapical approaches.

The ongoing PARTNER trial (Placement of AoRTic TraNscathetER Valve Trial) in the US is the first to randomise patients. In Cohort $B$ inoperable patients are randomised to TAVI or medical therapy, whereas in Cohort A patients with high operative risk are randomised to SAVR or TAVI. The trial completed its randomisation in early 2009. One-year outcome results will be reported in the forthcoming months. By study design, findings will only apply to this highly selected patient cohort, representing only a fraction of the global AS burden.

For a new technology to be accepted as a new asset in the armamentarium for treating symptomatic AS several essential questions need to be answered: Does the technology work? Which patients are likely to benefit (patient selection)? How does this new strategy compare with the alternatives? And what is the cost of the intervention? The proof of concept has been validated. The innovative less invasive transcatheter strategy should be at least as effective but safer than traditional SAVR or have proof of superiority for both safety and efficacy compared with medical therapy.

While anticipating the results of the PARTNER US trial, more than 18,000 patients worldwide had been treated with TAVI by May 20I0. Currently, TAVI is restricted to elderly patients who are considered at very high risk for conventional surgery, but unavoidably, with increased operator experience and access to the device, physicians will shift their attention to younger patients with a less pronounced operative risk. Similarly to what happened in the coronary revascularisation arena ${ }^{19}$ the blending of surgical and interventional expertise has created unique interdisciplinary dynamics reinforcing these new endeavours and paving the way for a randomised trial comparing TAVI with SAVR in a surgical moderate to high-risk patient population.

In high-risk patients, TAVI meets the criteria of ease of insertion, safety and excellent orifice area, but for lowerrisk patients a new randomised trial is necessary. The objective of the so-called SURTAVI trial is to assess whether, in patients with symptomatic severe aortic stenosis and at intermediate risk, TAVI is non-inferior to SAVR with respect to the event-free survival time of the combined endpoint of all-cause mortality and stroke at a median follow-up duration of two years. Ultimately these patients need to be followed for a longer time to determine long-term durability of these valves.

A secondary objective is to compare patients with symptomatic severe aortic stenosis and at intermediate risk treated with TAVI to SAVR with respect to quality of life, clinical benefit and health economics.

The interdisciplinary approach with co-operation between surgeons, cardiologists and anaesthetists, the so-called heart team, is crucial in this trial. This team will decide whether patients with intermediate risk can be randomised. If in the opinion of the heart team the surgical risk is deemed low, the patient will be operated on. In cases where the interdisciplinary team judges the risk too high for surgery the patient will receive TAVI, and for those patients where there is doubt which treatment offers the best outcome the patient will be randomised. This SURTAVI trial is likely to start in $20 \mathrm{II}$.

Theoretical benefits of these transcatheter instrumentations in a beating heart avoiding the need for musculoskeletal incisions, cardioplegic arrest, aortic cross clamping, full cardiopulmonary bypass (including subsequent LV septal motion abnormality) seem evident. Ultimately cost-effectiveness will determine whether the new treatment strategy is a valid option to be considered for reimbursement by governmental health institutions. The price tag of the device is essential and will ideally cover the company's capital investment made during research and development. The cost-effectiveness relationship will only become favourable once competitive companies enter the market and introduce alternative devices at lower prices. Randomised trials that address these issues are needed now.

\section{REFERENCES}

I lung B, Baron G, Butchart EG et al. A prospective survey of patients with valvular heart disease in Europe: the Euro Heart Survey on Valvular Heart Disease. Eur Heart J 2003; 24:I23I-43. doi:I0.I0I6/S0I95-668X(03)0020I-X
2 Van Geldorp MW, van Gameren M, Kappetein AP et al.Therapeutic decisions for patients with symptomatic severe aortic stenosis: room for improvement? Eur J Cardiothorac Surg 2009; 35:953-7. doi:I0.1016/j.ejcts.2009.01.043 
3 Rosenhek R, Zilberszac R, Schemper $M$ et al. Natural history of very severe aortic stenosis. Circulation 2010; I21:151-6.doi:10.1161/ CIRCULATIONAHA. 109.894170

4 Agarwal A, Kini AS, Attanti S et al. Results of repeat balloon valvuloplasty for treatment of aortic stenosis in patients aged 59 to 104 years. Am J Cardiol 2005; 95:43-7. doi:10.1016/j. amjcard.2004.08.06I

5 Bonow RO, Carabello BA, Chatterjee $K$ et al. 2008 Focused update incorporated into the ACC/AHA 2006 guidelines for the management of patients with valvular heart disease: a report of the American College of Cardiology/American Heart Association Task Force on Practice Guidelines (Writing Committee to Revise the 1998 Guidelines for the Management of Patients With Valvular Heart Disease): endorsed by the Society of Cardiovascular Anesthesiologists, Society for Cardiovascular Angiography and Interventions, and Society of Thoracic Surgeons. Circulation 2008; I I8:e523-66I. doi:I0.I I6I/CIRCULATIONAHA.108.190748

6 Vahanian A, Baumgartner H, Bax J et al. Guidelines on the management of valvular heart disease: the task force on the management of valvular heart disease of the European Society of Cardiology. Eur Heart J 2007; 28:230-68.

7 lung B, Cachier A, Baron G et al. Decision-making in elderly patients with severe aortic stenosis: why are so many denied surgery? Eur Heart J 2005; 26:27I4-20. doi:10.1093/eurheartj/ehi471

8 Bouma BJ, van den Brink RB, van der Meulen JH et al. To operate or not on elderly patients with aortic stenosis: the decision and its consequences. Heart 1999; 82:143-8.

9 Webb JG, Pasupati S, Humphries K et al. Percutaneous transarterial aortic valve replacement in selected high-risk patients with aortic stenosis. Circulation 2007; ||6:755-63. doi:10.|16|| CIRCULATIONAHA. 107.698258

10 Grube E, Laborde JC, Gerckens $\mathrm{U}$ et al. Percutaneous implantation of the CoreValve self-expanding valve prosthesis in high-risk patients with aortic valve disease: the Siegburg first-in-man study. Circulation 2006; I 14:1616-24. doi:I0. I I6I/CIRCULATIONAHA. 106.639450
II Webb JG, Altwegg L, Boone RH et al. Transcatheter aortic valve implantation: impact on clinical and valve-related outcomes. Circulation 2009; I 19:3009-16. doi:I0.1 I6I/CIRCULATIONAHA. I08.837807

12 Piazza N, Grube E, Gerckens U et al. Procedural and 30-day outcomes following transcatheter aortic valve implantation using the third generation (I8 Fr) corevalve revalving system: results from the multicentre, expanded evaluation registry I-year following CE mark approval. Eurolntervention 2008; 4:242-9.

I3 Brown JM, O'Brien SM, Wu C et al. Isolated aortic valve replacement in North America comprising 108,687 patients in 10 years: changes in risks, valve types, and outcomes in the Society of Thoracic Surgeons National Database. J Thorac Cardiovasc Surg 2009; I37:82-90. doi:I0.1016/j.jtcvs.2008.08.0I5

14 Halkos ME, Chen EP, Sarin EL et al. Aortic valve replacement for aortic stenosis in patients with left ventricular dysfunction. Ann Thorac Surg 2009; 88:746-5I. doi:I0.1016/j.athoracsur.2009.05.078

15 Kolh P, Kerzmann A, Honore $\mathrm{C}$ et al. Aortic valve surgery in octogenarians: predictive factors for operative and long-term results. Eur J Cardio-thoracic Surg 2007; 31:600-6. doi:10.1016/j. ejcts.2007.01.003

16 Walther T, Schuler G, Borger MA et al. Transapical aortic valve implantation in 100 consecutive patients: comparison to propensity-matched conventional aortic valve replacement. Eur Heart J 2010; 31:1398-403. doi:10.1093/eurheartj/ehq060

17 Thomas M, Schymik G, Walther T et al. Thirty-day results of the SAPIEN aortic bioprosthesis European Outcome (SOURCE) Registry: a European registry of transcatheter aortic valve implantation using the Edwards SAPIEN valve. Circulation 2010; I22:62-9. doi: I0.1 I6I/CIRCULATIONAHA. 109.907402

I8 Piazza N, Cutlip DE, OnumaY et al. Clinical endpoints in transcatheter aortic valve implantation: a call to ARC for standardised definitions. Eurolntervention 2009; 5:29-3I. doi: I0.4244/EIJV5IIA5

19 Serruys PW. Keynote address-EuroPCR 2008, Barcelona, May 14th, 2008. Transcatheter aortic valve implantation: state of the art. Eurolntervention 2009; 4:558-65. doi: I0.4244/EIJV4I5A95

\section{CONTINUING MEDICAL EDUCATION (CME)}

\section{Online distance learning and self-assessment from the RCPE}

To allow users to update their general medical knowledge, the online CME modules contain a variety of material, including articles, interactive cases and quizzes, webstreamed lectures and self-assessment multiple choice questions (MCQs). Each module also includes material of particular relevance to those undertaking core medical training.
CME modules are eligible for external distancelearning Continuing Professional Development (CPD) credits under the UK Federation of Royal Colleges of Physicians CPD scheme. To claim these credits you must take the MCQ test for the module (only available online). Full instructions are given with each module.
The College's online CME programme is available to Fellows, Collegiate Members, Associates and e-Associates. Log on to the Fellows' and Members' secure area at: http://www.rcpe.ac.uk. If you have forgotten your username or password please contact: webmaster@rcpe.ac.uk
Currently available for CPD points

- Oncology

- Cardiology

\section{Coming soon}

- Neurology

- Rheumatology

- Tropical Medicine

- Respiratory 